\title{
Reprocessable, self-adhesive, and recyclable carbon fiber reinforced composites using a catalyst-free self-healing bio-based vitrimer matrix
}

Yazhou $\mathrm{Xu}^{\mathrm{a}, \mathrm{b}}$, Songlin Dai ${ }^{\mathrm{a}}$, Haibo Zhang ${ }^{\mathrm{a} * *}$, Liangwu $\mathrm{Bi}^{\mathrm{a}, \mathrm{c}}$, Jianxin Jiang ${ }^{\mathrm{b}}$, Yuxiang

$$
\text { Chen }^{\mathrm{a}, \mathrm{c}, *}
$$

${ }^{a}$ Institute of Chemical Industry of Forest Products, Chinese Academy of Forestry, National Engineering Laboratory for Biomass Chemical Utilization, Nanjing 210042,

China.

${ }^{b}$ College of Materials Science and Technology, Beijing Forestry University, Beijing, 100083, China.

${ }^{c}$ Co-Innovation Center of Efficient Processing and Utilization of Forest Resources, Nanjing Forestry University, Nanjing 210037, China.

*Corresponding Authors

Email: shdzhanghaibo@163.com (Haibo Zhang)

Email: cyxlhs@126.com (Yuxiang Chen)

The supporting information includes 10 pages and 10 figures. 
The specific preparation processes (Figure S1) of TOTGE are as follows:

1. Preparation of fatty acid methyl esters

Tung oil (300.00 g), methanol (80.00 g) and sodium hydroxide $(\mathrm{NaOH}, 2.10 \mathrm{~g})$ were reacted in a flask for $2 \mathrm{~h}$ at $70^{\circ} \mathrm{C}$. After the reaction, the yellow liquid in the upper layer was dissolved in ethyl acetate and the mixture was washed with saturated salt water to neutral. Fatty acid methyl ester $(256.65 \mathrm{~g})$ was obtained, after removal of ethyl acetate.

\section{Preparation tung maleic acid methyl ester}

The Diels-Alder reaction of fatty acid methyl ester (300.00 g) and maleic anhydride (203.00 g) occurred at $140^{\circ} \mathrm{C}$ for $3 \mathrm{~h}$. Subsequently, an excess of aqueous $\mathrm{NaOH}$ was added to the mixture to ensure that the anhydride ring was completely opened. Ethyl acetate was added to dissolve the fatty acid methyl ester that did not undergo Diels-Alder reaction. The lower yellow liquid layer was taken, acidified and the organic layer was retained, then the organic layer was dissolved in ethyl acetate and washed with saturated salt water to neutral. Tung maleic acid methyl ester ( $270.00 \mathrm{~g})$ was obtained after removing ethyl acetate with an acid value of $270.35 \mathrm{mg} / \mathrm{g}$ (theoretical acid value of $275.00 \mathrm{mg} / \mathrm{g}$ ).

\section{Preparation of tung maleic triacid (TMTA)}

Tung maleic acid methyl ester $50.00 \mathrm{~g}$ ) dissolved in ethanol was hydrolyzed by an excess of aqueous $\mathrm{NaOH}$ solvent at $90^{\circ} \mathrm{C}$. After the reaction, the aqueous layer was taken and acidified, then washed with saturated saline to neutral, and TMTA (43.50 g) was obtained, which had an acid value of $381.50 \mathrm{mg} / \mathrm{g}$ (theoretical acid value of $427.16 \mathrm{mg} / \mathrm{g})$. Its ${ }^{1} \mathrm{H}$ NMR and ${ }^{13} \mathrm{C}$ NMR are shown in Figure $\mathrm{S} 1$. 


\section{Preparation of TOTGE}

TMTA $(50.00 \mathrm{~g})$ was reacted with epichlorohydrin $(314.00 \mathrm{~g})$ for $4 \mathrm{~h}$ at $117^{\circ} \mathrm{C}$. Subsequently, $\mathrm{NaOH}(13.65 \mathrm{~g})$ and calcium oxide (19.00 g) were added to the mixture at $60^{\circ} \mathrm{C}$ and the reaction was continued for $3 \mathrm{~h}$. When the reaction was finished, TOTGE (53.80 g) was obtained, after removing the excess epichlorohydrin. It had an epoxy value of $0.43 \mathrm{~mol} / 100 \mathrm{~g}$ (theoretical epoxy value of $0.53 \mathrm{~mol} / 100 \mathrm{~g}$ ).

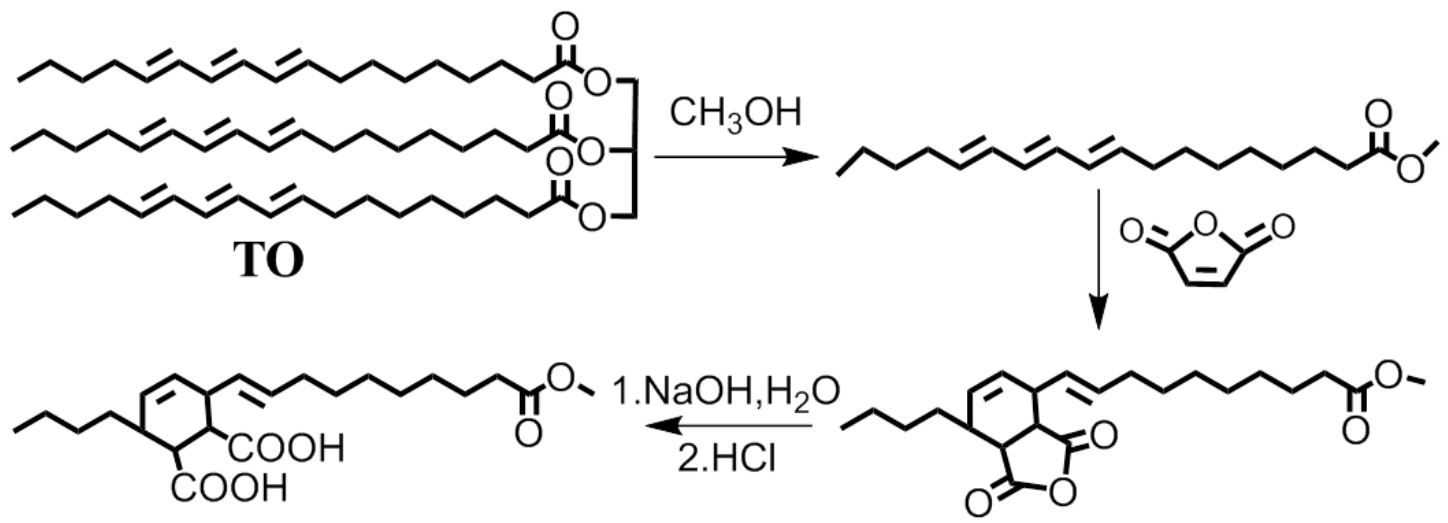

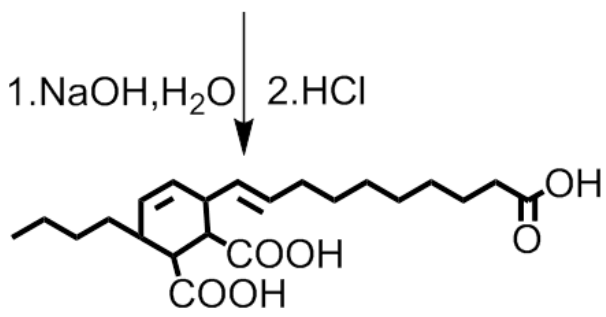

TMTA

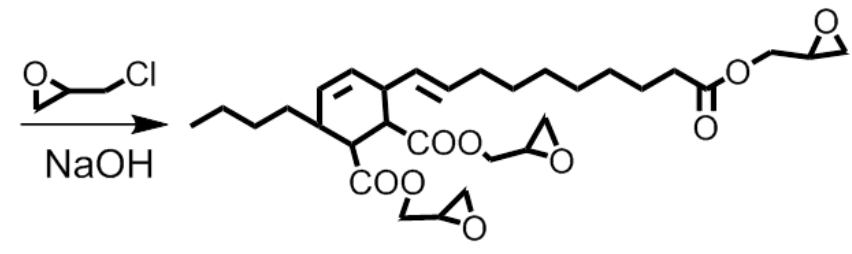

TOTGE

Figure S1. Preparation of TOTGE. 

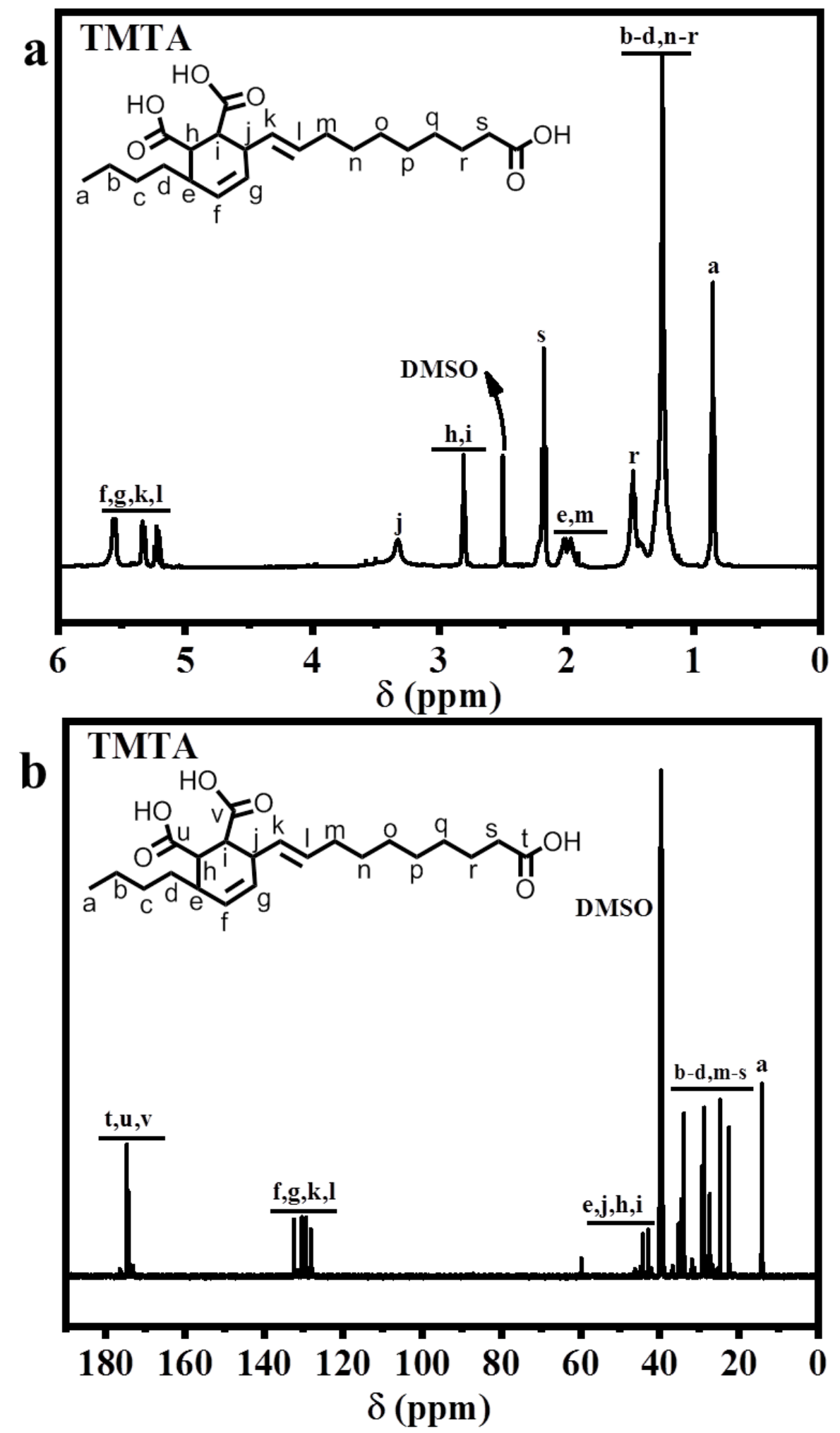

Figure S2. ${ }^{1} \mathrm{H}$ NMR and ${ }^{13} \mathrm{C}$ NMR spectrum of TMTA. 

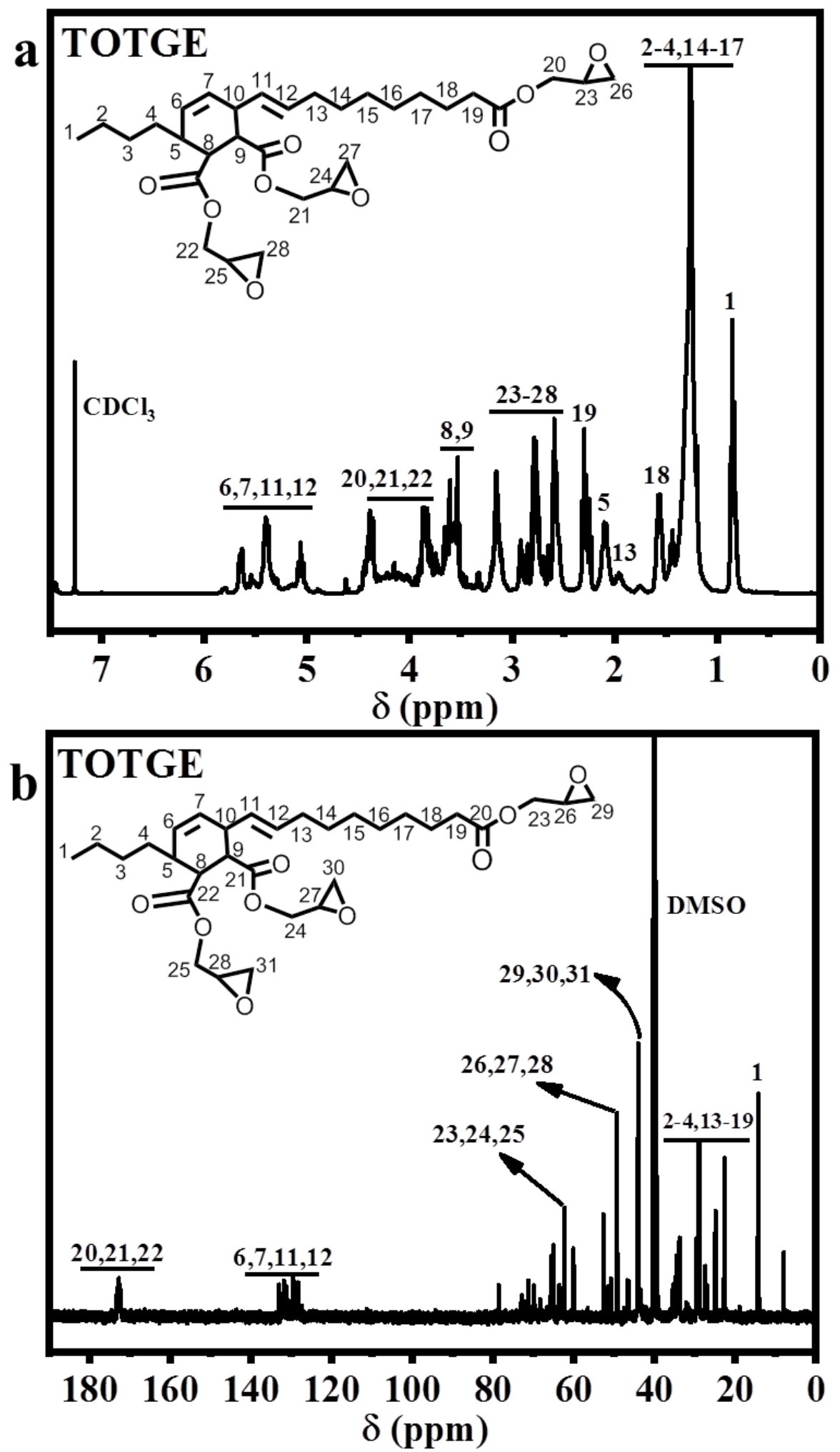

Figure S3. ${ }^{1} \mathrm{H}$ NMR and (b) ${ }^{13} \mathrm{C}$ NMR spectra of TOTGE. 

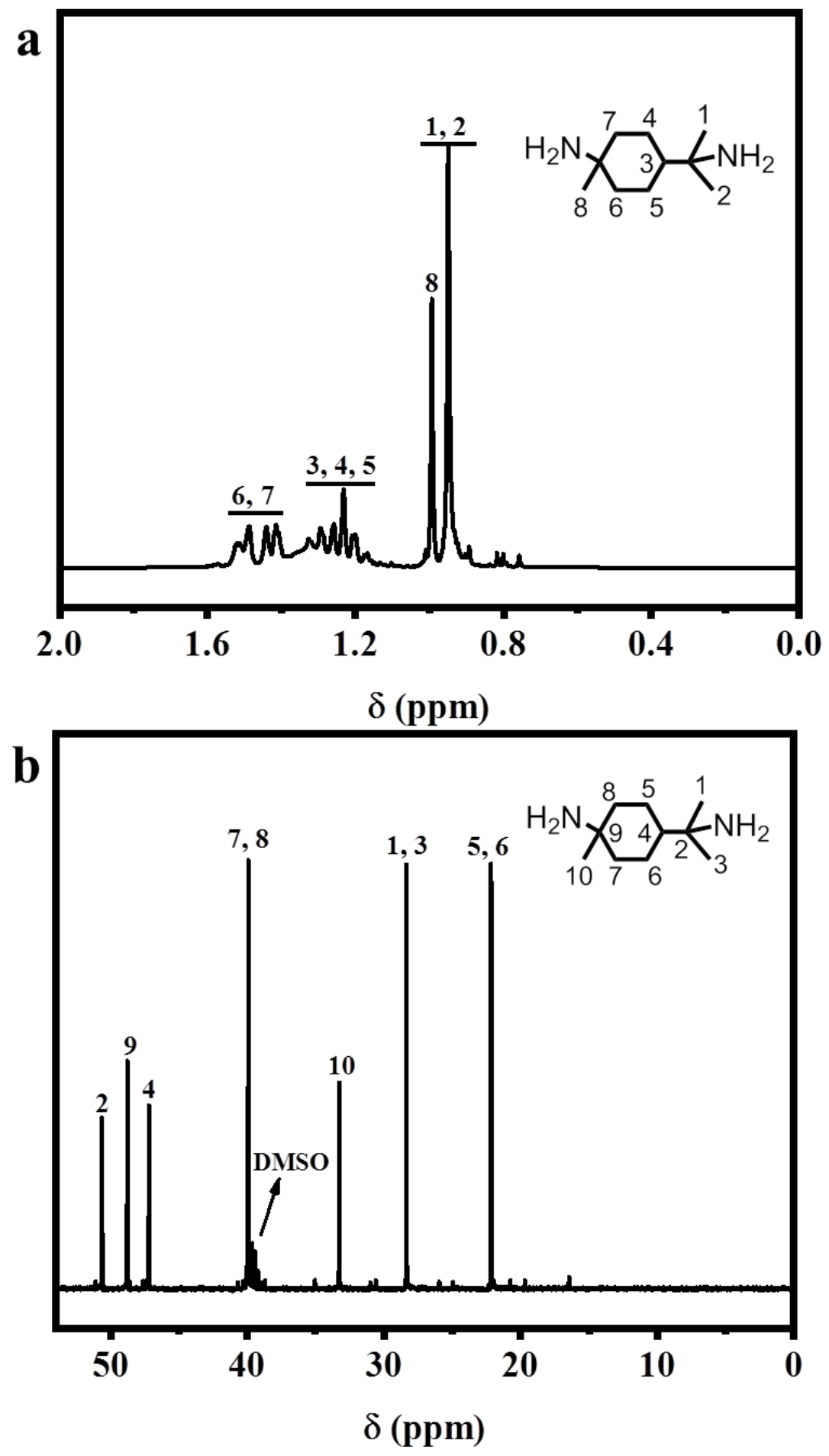

Figure S4. ${ }^{1} \mathrm{H}$ NMR and ${ }^{13} \mathrm{C}$ NMR spectrum of MDA. 

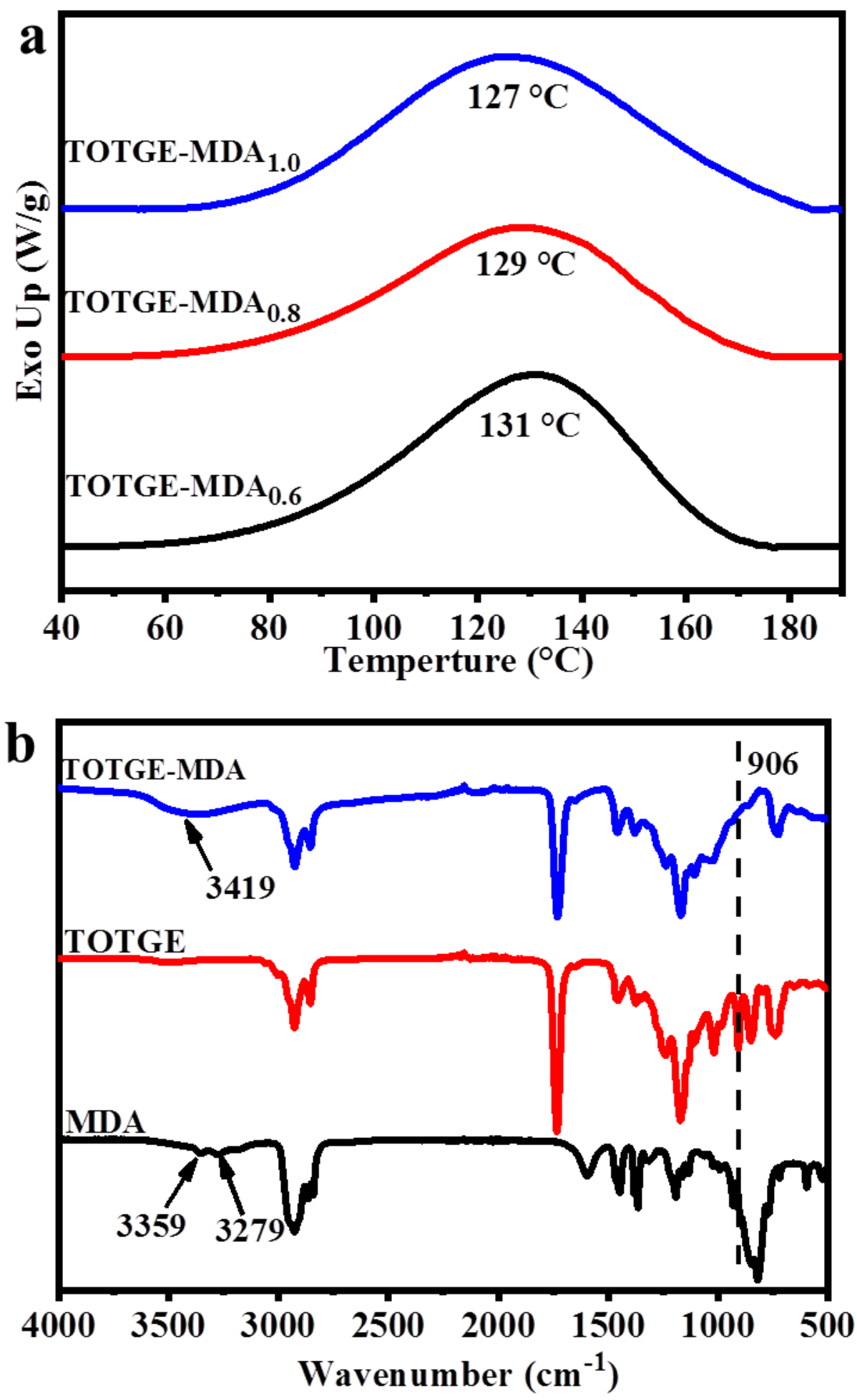

Figure S5. (a) Differential scanning calorimetry curves for the mixtures of TOTGE and MDA at a heating rate of $10^{\circ} \mathrm{C} \mathrm{min}^{-1}$. (b) FTIR spectra of MDA, TOTGE, and TOTGE-MDA. 


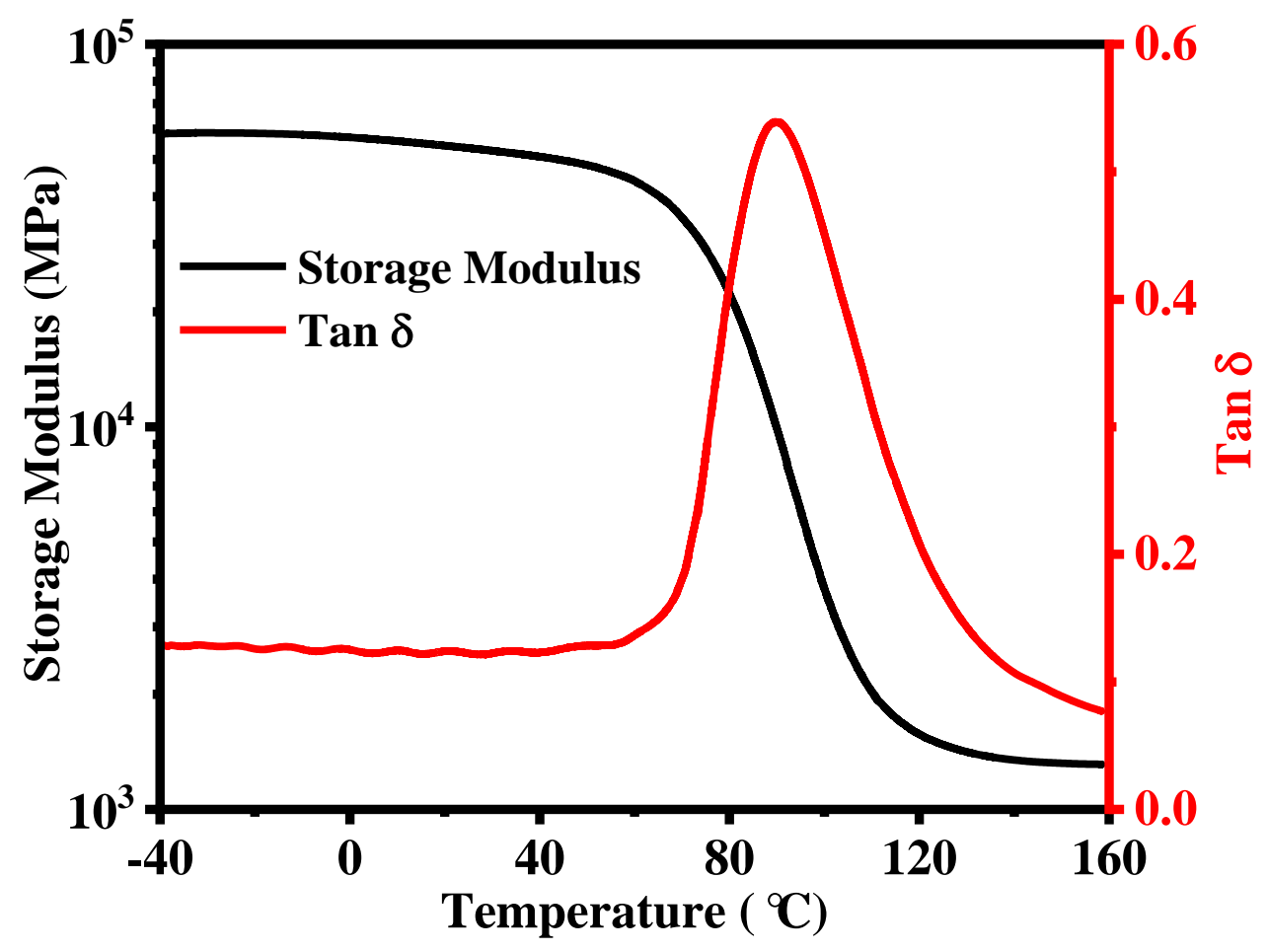

Figure S6. Storage modulus and $\tan \delta$ curves of the TOTGE-MDA-CF composite.

The specific purification process of the degradation products is as follows:

After the TOTGE-MDA matrix was degraded in EA, $10 \mathrm{~mL}$ of the degradation mixture was dissolved in ethyl acetate and washed five times with saturated salt water to ensure removal of the water-soluble EA and degradation product B (Figure 14). After removal of ethyl acetate, the degradation product A was obtained.

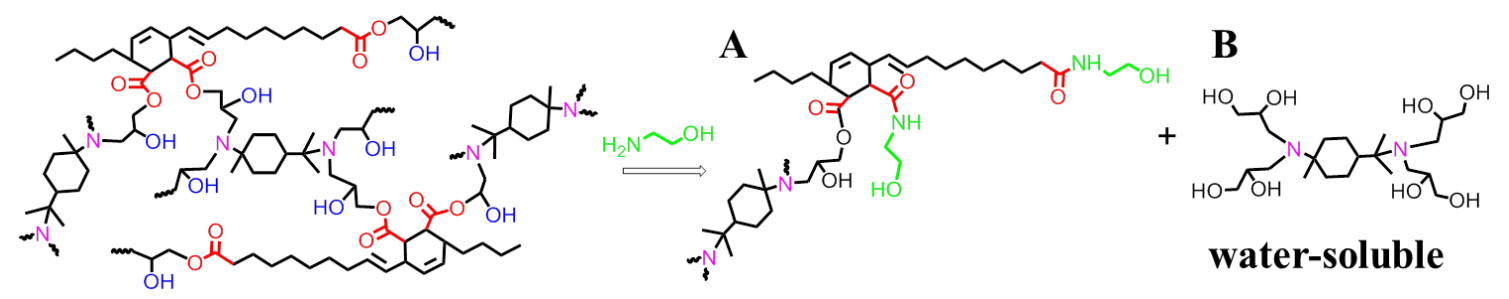

Figure S7. Degradation reaction of TOTGE-MDA-CF composite matrix. 


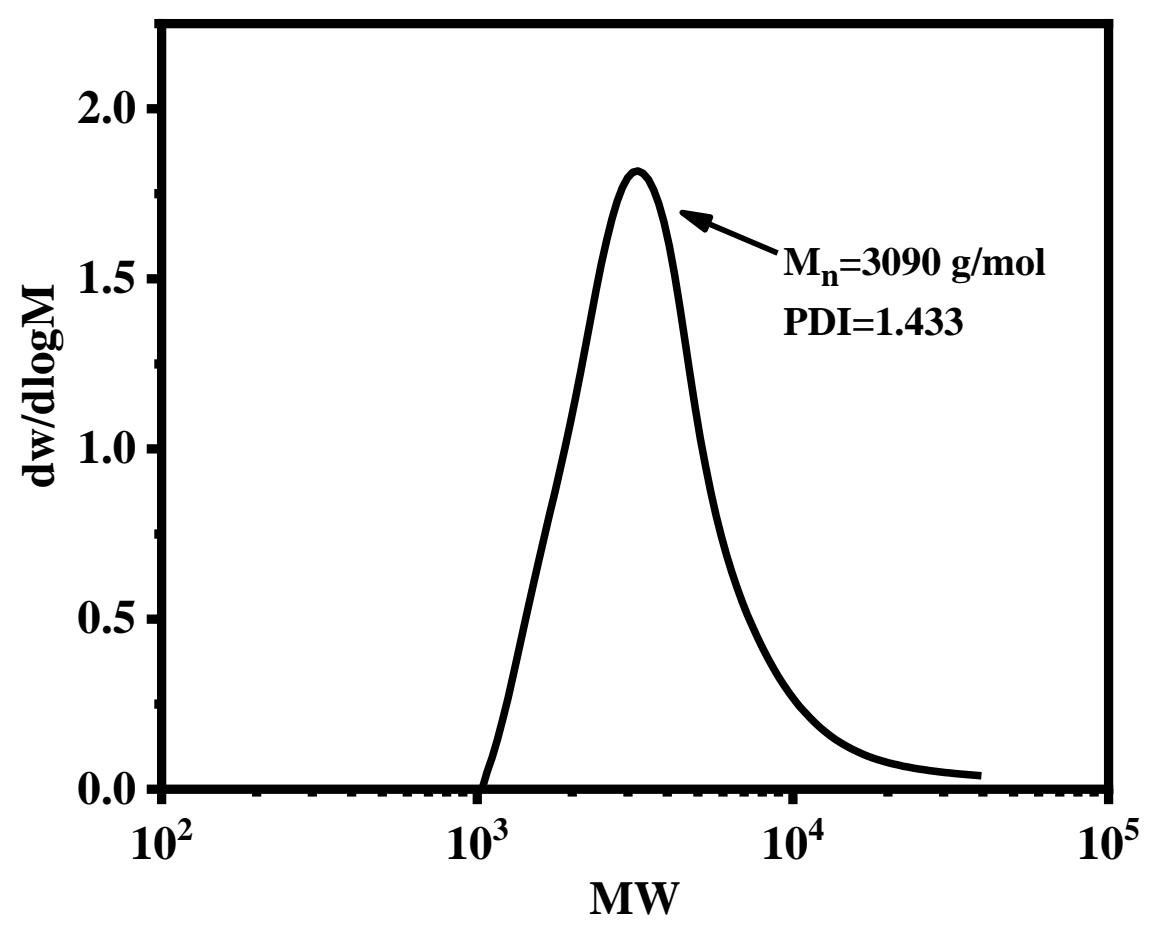

Figure S8. GPC of degradation product A.

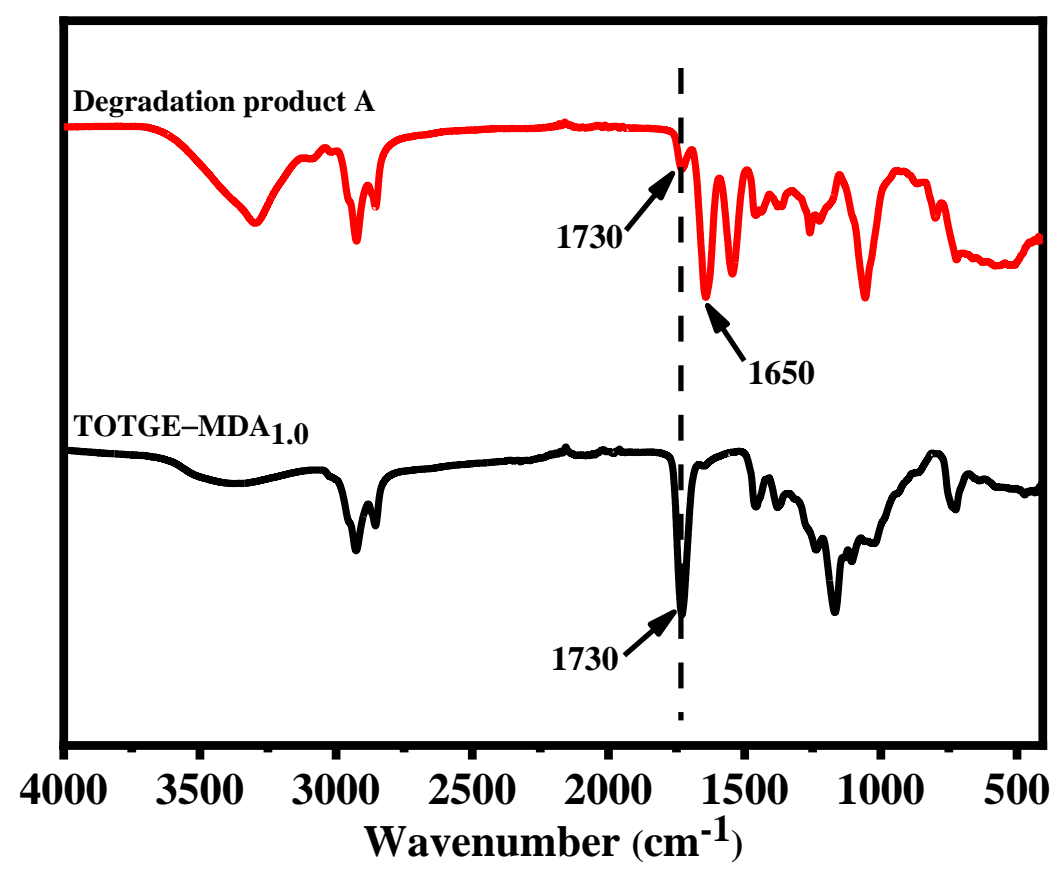

Figure S9. FTIR spectra of TOTGE-MDA 1.0 matrix and degradation product A. 


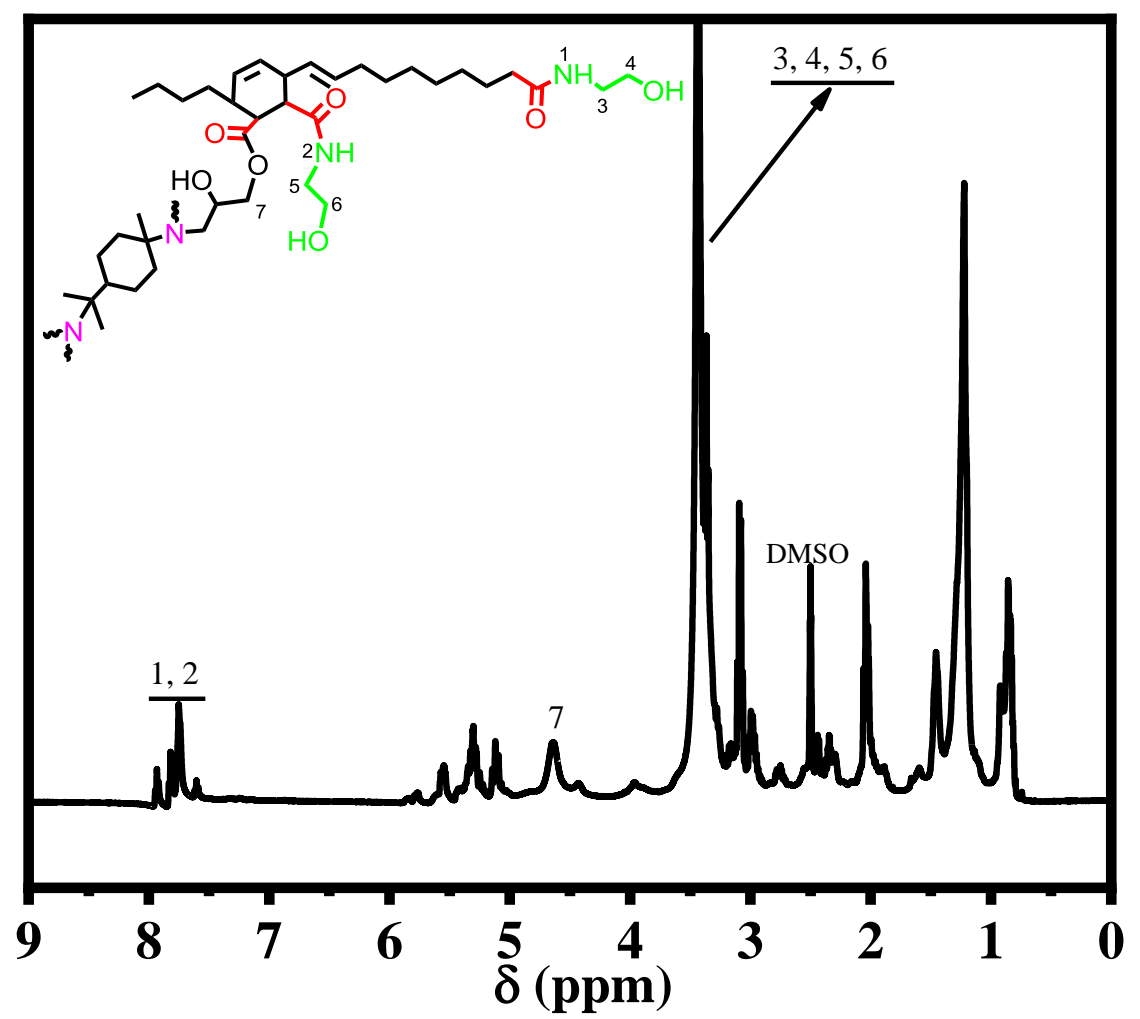

Figure S10. ${ }^{1} \mathrm{H}$ NMR spectra of the degradation product A. 